\title{
Automated Data Quality Assessment in Electron Cryomicroscopy
}

\author{
Htet Khant, Steven Ludtke, Wah Chiu
}

National Center for Macromolecular Imaging, Verna and Marrs McLean Department of Biochemistry and Molecular Biology, Baylor College of Medicine, One Baylor Plaza, Houston, TX 77030, USA

Data quality in electron cryomicroscopy is critical to achieve high resolution structures of macromolecular assemblies. Historically, data quality is evaluated after the completion of microscopy experiment. In recent years, charge coupled device (CCD) camera becomes available in electron cryomicroscopy. This allows instant feedback on the data quality, which is dependent upon specimen, microscope conditions and ambient environment. However, it is quite overwhelming to do manual comprehensive assessment of every CCD image at a typical data acquisition rate for an entire microscopy session. Therefore, we are developing a program that can automatically assess the data quality as well as imaging parameters during a microscopy session.

Our Data Quality Assessment Program stream-lines the data quality evaluation through MATLAB's graphical user interfaces, which integrates our existing software packages, JAMES and EMAN (fig. 1). JAMES is a python-based program for data collection, which communicates with microscope and CCD camera [1]. EMAN is a single particle reconstruction package [2]. The program reads CCD images acquired by JAMES and calls EMAN's $\mathrm{C}^{++}$programs to parameterize contrast transfer function (CTF). First, CCD image is filtered to remove extraneous bright and dark pixels resulted from x-rays and dead CCD pixels. Then the filtered image is split into a stack of smaller images, from which EMAN determines parameters for CTF and associated decay functions. Once these parameters have been determined, the program displays the raw CCD image, the image stack's 2-D and rotationally averaged power spectra (figs. 2A-C). In addition, it also plots experimental Bfactors versus true defocus values for the images that have been collected (fig. 2D). Our Data Quality Assessment Program, JAMES and EMAN can be implemented on a multi-processors PC for routine use, where computations can be assigned to different processors for better performance. We tested our program using various single particle cryo specimens imaged on JEM2010F microscope equipped with 4k x 4k Gatan CCD camera under low dose conditions.

Our Data Quality Assessment Program provides the microscope user with an opportunity and convenience to make necessary adjustments while data is being collected. It also assure data quality needed to achieve high resolution structure by electron cryomicroscopy.

\section{References}

[1] Booth CR, J. W., Baker ML, Zhou ZH, Ludtke SJ, Chiu W. (2004). "A 9 angstroms single particle reconstruction from CCD captured images on a $200 \mathrm{kV}$ electron cryomicroscope." J Struct Biol 147(2): 116-27.

[2] Ludtke SJ, J. J., Song JL, Chuang DT, Chiu W. (2001). "A 11.5 A single particle reconstruction of GroEL using EMAN." J Mol Biol 314(2): 253-62.

[3] Supported by P41RR02250. 


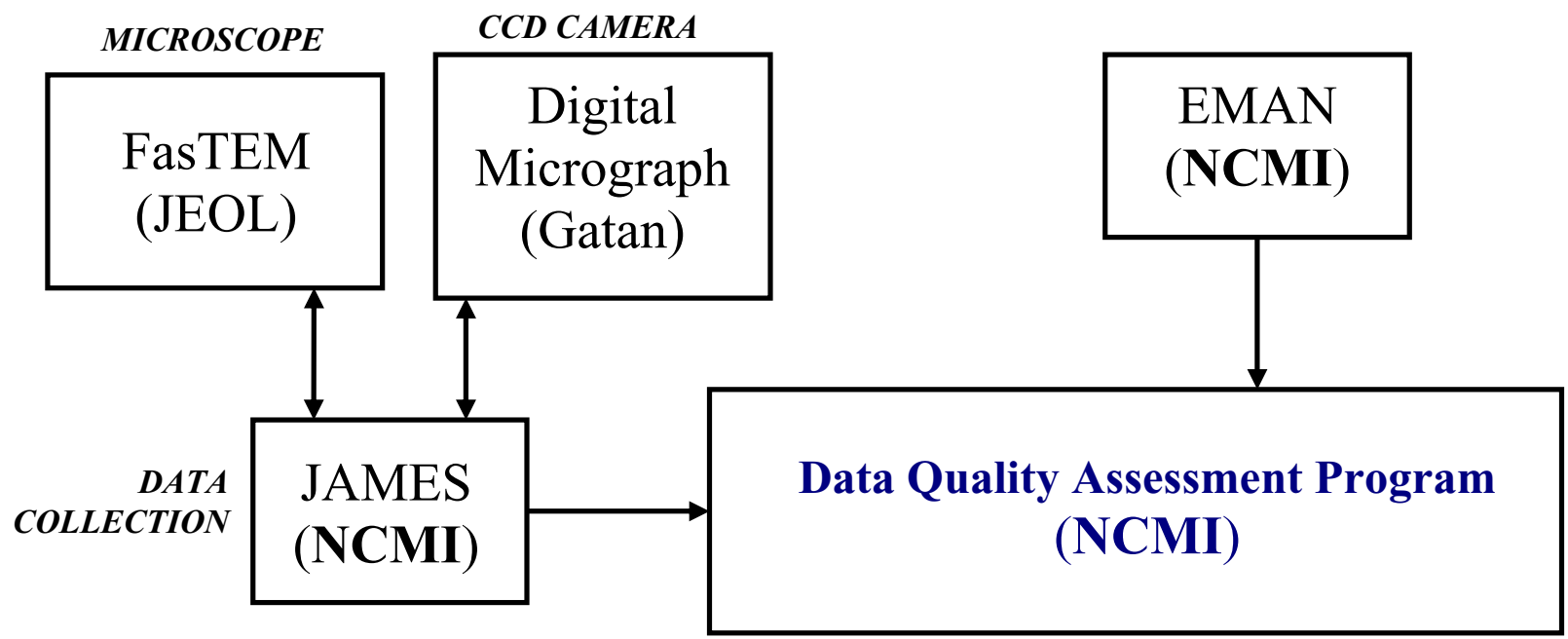

Figure 1. Data flow between Data Quality Assessment Programs and various programs. FasTEM operates microscope and Digital Micrograph controls CCD camera. JAMES communicates with both FasTEM and Digital Micrograph for CCD image acquisition.
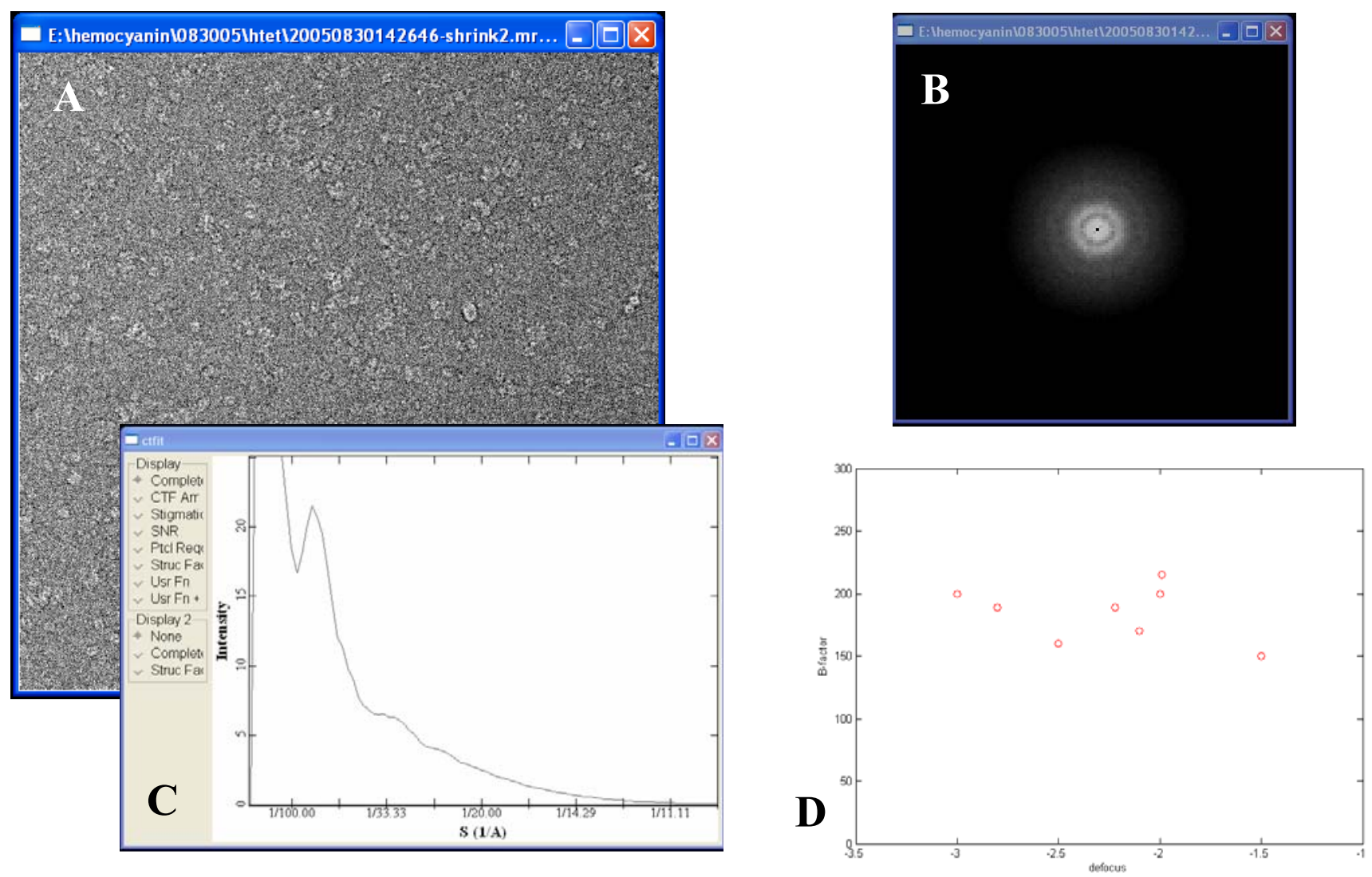

Figure 2. Example snapshots of program's outputs. (A) CCD image of ice-embedded particles (B) Computed FFT of boxed out particle images (C) Radial power spectrum (D) Continuously updated $B$ factor plot of the images in a microscope session. 